\title{
Frequency and 2D Angle Estimation Based on a Sparse Uniform Array of Electromagnetic Vector Sensors
}

\author{
Fei Ji ${ }^{1}$ and Sam Kwong ${ }^{2}$ \\ ${ }^{1}$ School of Electronic and Information Engineering, South China University of Technology, Guangzhou 510640, China \\ ${ }^{2}$ Department of Computer Science, City University of Hong Kong, Tat Chee Avenue, Kowloon, Hong Kong
}

Received 25 April 2005; Revised 25 January 2006; Accepted 29 January 2006

Recommended for Publication by Joe C. Chen

\begin{abstract}
We present an ESPRIT-based algorithm that yields extended-aperture two-dimensional (2D) arrival angle and carrier frequency estimates with a sparse uniform array of electromagnetic vector sensors. The ESPRIT-based frequency estimates are first achieved by using the temporal invariance structure out of the two time-delayed sets of data collected from vector sensor array. Each incident source's coarse direction of arrival (DOA) estimation is then obtained through the Poynting vector estimates (using a vector crossproduct estimator). The frequency and coarse angle estimate results are used jointly to disambiguate the cyclic phase ambiguities in ESPRIT's eigenvalues when the intervector sensor spacing exceeds a half wavelength. Monte Carlo simulation results verified the effectiveness of the proposed method.
\end{abstract}

Copyright ( 2006 Hindawi Publishing Corporation. All rights reserved.

\section{INTRODUCTION}

The localization of source signals using vector sensor data processing has attracted significant attentions lately. Many advantages of using the vector sensor array have been identified and many array data processing techniques for source localization and polarization estimation using vector sensors have been developed. Nehorai and Paldi developed the Cramér-Rao bound (CRB) and the vector cross-product DOA estimator using the vector cross product of the electricfield and the magnetic-field vector estimates $[1,2]$. Li [3] developed ESPRIT-based angle and polarization estimation algorithm using an arbitrary array with small loops and short dipoles. Identifiablity and uniqueness study associated with vector sensors were done by Hochwald and Nehorai [4], Ho et al.[5] and Tan et al. [6]. Hochwald and Nehorai [7] studied parameter estimations with application to remote sensing by vector sensors. Ho et al. [8] developed a high-resolution ESPRIT-based method for estimating the DOA of partially polarized sources. Ho et al. [9] further studied the DOA estimation with vector sensors for scenarios where completely and incompletely polarized signals may coexist. Wong [10] has showed that the vector cross-product DOA estimator remains fully applicable for a pair of dipole triad and loop triad spatially displaced by an arbitrary and unknown distance (rather than being collocated). Uni-vector-sensor ESPRIT is first presented to estimate $2 \mathrm{D} \mathrm{DOA}$ and the polarization states of multiple monochromatic noncoherent incident sources using a single electromagnetic vector sensor by Wong and Zoltowski [11]. Nehorai and Tichavsky [12] presented an adaptive cross-product algorithm for tracking the direction to a moving source using an electromagnetic vector sensor. Ko et al. [13] proposed a structure for adaptively separating, enhancing, and tracking up to three uncorrelated broadband sources with an electromagnetic vector sensor. Wong [14] proposed an ESPRIT-based adaptive geo-location and blind interference rejection scheme for multiple noncooperative wideband fast frequency-hop signals using one electromagnetic vector sensor. The maximum likelihood (ML) and minimum variance distortionless response (MVDR) estimators for signal DOA and polarization parameters for correlated sources are derived by Rahamim et al. [15]. In addition, a novel preprocessing method based on the polarization smoothing algorithm (PSA) for "decorrelating" the signals was also presented. Wong and Zoltowski [16] presented a self-initiating MUSIC-based DOA and polarization estimation algorithm for an arbitrarily spaced array of identically oriented electromagnetic vector sensors. Their proposed algorithm is able to exploit the incident sources' polarization diversity and to decouple the estimation of the sources' 
arrival angles from the estimation of the sources' polarization parameters. The same authors further developed a closedform direction-finding algorithm applicable to multiple arbitrarily spaced vector sensors at possibly unknown locations [17]. A sparse uniform array suffers cyclic ambiguity in its direction-cosine estimates due to the spatial Nyquist sampling theorem. Zoltowski and Wong then further presented another novel ESPRIT-based 2D arrival angle estimation scheme to resolve the aforementioned ambiguity and achieve aperture extension for a sparse uniform array of vector sensors spaced much further apart than a half wavelength [18]. An improved version of the disambiguation algorithm is also presented in [19].

In fact, frequency estimation is a fundamental problem in estimation theory and its applications include radar, array signal processing, and frequency synchronization. For scalar sensor array, a number of ESPRIT-based angle and frequency estimation methods have been proposed. Lemma et al. presented joint angle-frequency estimation method using multidimensional and multiresolution ESPRIT algorithms [20, 21]. Zoltowaki and Mathews discuss ESPRIT-based realtime angle-frequency estimation algorithm using scalar sensor array [22].

In this paper, we try to combine the ESPRIT-based frequency estimation with Wong's ESPRIT-based 2D DOA estimation scheme in [18] to yield extended-aperture twodimensional (2D) arrival angle and carrier frequency estimates with a sparse uniform array of electromagnetic vector sensors. Most of the works mentioned above have previously proposed direction-finding and polarization estimation algorithms using electromagnetic vector sensors; however, this paper is the first in advancing an algorithm for the estimation of both arrival angles and arrival delays.

In the newly proposed algorithm, the ESPRIT-based frequency estimates are achieved using the temporal invariance structure out of two time-delayed sets of data collected from vector sensor array. In that each incident source's direction of arrival (DOA) coarse estimation is obtained through a vector cross-product estimator. Then the frequency estimates and coarse angle estimates results are used jointly to disambiguate the cyclic phase ambiguities in ESPRIT's eigenvalues when the intervector sensor spacing exceeds a half wavelength.

\section{MATHEMATICAL MODEL}

Consider the scenario of $K$ uncorrelated monochromatic completely polarized transverse electromagnetic planewaves signals with different carrier frequencies, impinging on an $L$-shaped array of regularly equally spaced and identical electromagnetic vector sensors from directions $\left(\theta_{k}, \phi_{k}\right)$ and polarization parameters $\left(\gamma_{k}, \eta_{k}\right)(k=1, \ldots, K) .0 \leq \theta_{k}<\pi$ is the $k$ th signal's elevation angle measured from vertical $z$ axis, $0 \leq \phi_{k}<2 \pi$ is azimuth angle, $0 \leq \gamma_{k}<\pi / 2$ is auxiliary polarization angle, and $-\pi \leq \eta_{k}<\pi$ is polarization phase difference.

The signal source model is given by

$$
s_{k}(n)=\sqrt{\mathbf{P}_{k}} e^{j\left(2 \pi f_{k} n+\varphi_{k}\right)}, \quad n=1,2, \ldots, N,
$$

where $\mathbf{P}_{k}$ is the $k$ th source's energy, $\varphi_{k}$ is the $k$ th signal's uniformly distributed random phase, and $N$ is the number of independent samples collected by the array. $f_{k}$ is the $k$ th source's digital frequency (between -0.5 and 0.5 ) normalized to the sampling frequency $F_{s}$ which satisfies the Nyquist sampling theorem for all the signals' frequencies. Here we normalize to $F_{s}=1$.

A vector sensor contains three electric and three magnetic orthogonal sensors. The spatial response in matrix notation of one vector sensor for the $k$ th signal may be expressed as follows [11]:

$$
\mathbf{g}_{k} \stackrel{\text { def }}{=}\left[\begin{array}{c}
e_{x k} \\
e_{y k} \\
e_{z k} \\
h_{x k} \\
h_{y k} \\
h_{z k}
\end{array}\right] \stackrel{\text { def }}{=}\left[\begin{array}{c}
\sin \gamma_{k} \cos \theta_{k} \cos \phi_{k} e^{j \eta_{k}}-\cos \gamma_{k} \sin \phi_{k} \\
\sin \gamma_{k} \cos \theta_{k} \sin \phi_{k} e^{j \eta_{k}}+\cos \gamma_{k} \cos \phi_{k} \\
-\sin \gamma_{k} \sin \theta_{k} e^{j \eta_{k}} \\
-\cos \gamma_{k} \cos \theta_{k} \cos \phi_{k}-\sin \gamma_{k} \sin \phi_{k} e^{j \eta_{k}} \\
-\cos \gamma_{k} \cos \theta_{k} \sin \phi_{k}+\sin \gamma_{k} \cos \phi_{k} e^{j \eta_{k}} \\
\cos \gamma_{k} \sin \theta_{k}
\end{array}\right] .
$$

Note that $\mathbf{g}_{k}$ does not depend on the signal frequency. $\mathbf{e}_{k} \stackrel{\text { def }}{=}\left[e_{x k}, e_{y k}, e_{z k}\right]^{T}$ and $\mathbf{h}_{k} \stackrel{\text { def }}{=}\left[h_{x k}, h_{y k}, h_{z k}\right]^{T}$ (where the superscript $T$ denotes the vector transpose operator) are orthogonal to each other and the source's direction of propagation, that is, the normalized Poynting vector $\mathbf{p}_{k}$ [18],

$$
\mathbf{p}_{k}=\left[\begin{array}{c}
p_{x k} \\
p_{y k} \\
p_{z k}
\end{array}\right]=\frac{\mathbf{e}_{k}}{\left\|\mathbf{e}_{k}\right\|} \times \frac{\mathbf{h}_{k}^{*}}{\left\|\mathbf{h}_{k}\right\|}=\left[\begin{array}{c}
u_{k} \\
v_{k} \\
w_{k}
\end{array}\right]=\left[\begin{array}{c}
\sin _{\theta_{k}} \cos \phi_{k} \\
\sin _{\theta_{k}} \sin \phi_{k} \\
\cos _{\theta_{k}}
\end{array}\right],
$$

where $*$ denotes complex conjugation and $u_{k}, v_{k}, w_{k}$, respectively, symbolize the direction cosine along the $x$-axis, $y$-axis, and the $z$-axis.

The spatial phase factor of the $k$ th signal at the $m$ th vector sensor located $(m-1) \Delta$ along $x$-axis equals

$$
\begin{aligned}
q_{m}^{x}\left(\theta_{k}, \phi_{k}\right) & \stackrel{\text { def }}{=} e^{j 2 \pi f_{k} F_{s}(m-1) \Delta u_{k} / c} \\
& =e^{j 2 \pi f_{k} F_{s}(m-1) \Delta \sin \theta_{k} \cos \phi_{k} / c}, \quad m=1,2, \ldots, M,
\end{aligned}
$$

where $c$ is the velocity of light. The spatial phase factor of the $k$ th signal at the $l$ th vector sensor located $(l-1) \Delta$ along $y$-axis equals

$$
\begin{aligned}
q_{l}^{y}\left(\theta_{k}, \phi_{k}\right) & =e^{j 2 \pi f_{k} F_{s}(l-1) \Delta v_{k} / c} \\
& =e^{j 2 \pi f_{k} F_{s}(l-1) \Delta \sin \theta_{k} \sin \phi_{k} / c}, \quad l=1,2, \ldots, L .
\end{aligned}
$$

The $6 \times 1$ vector measurement in the $n$th snapshot is produced by the $m$ th vector sensor along $x$-axis and the $l$ th vector sensor along $y$-axis, respectively,

$$
\begin{aligned}
& \mathbf{z}_{m}^{x}(n)=\sum_{k=1}^{K} \mathbf{g}_{k} q_{m}^{x}\left(\theta_{k}, \phi_{k}\right) S_{k}(n)+\mathbf{n}_{m}^{x}(n), \\
& \mathbf{z}_{l}^{y}(n)=\sum_{k=1}^{K} \mathbf{g}_{k} q_{l}^{y}\left(\theta_{k}, \phi_{k}\right) S_{k}(n)+\mathbf{n}_{l}^{y}(n),
\end{aligned}
$$


where $\mathbf{n}_{m}^{x}(n)$ and $\mathbf{n}_{l}^{y}(n)$, respectively, symbol $6 \times 1$ complexvalued zero-mean additive white noise vector in $n$th snapshot at the $m$ th vector sensor along $x$-axis and the $l$ th vector sensor along $y$-axis.

Time-delayed data collected from the linear vector sensor array along $x$-axis is

$$
\begin{aligned}
\mathbf{z}_{m}^{x}\left(n+n_{0}\right) & =\sum_{k=1}^{K} \mathbf{g}_{k} q_{m}^{x}\left(\theta_{k}, \phi_{k}\right) S_{k}\left(n+n_{0}\right)+\mathbf{n}_{m}^{x}\left(n+n_{0}\right) \\
& =\sum_{k=1}^{K} \mathbf{g}_{k} q_{m}^{x}\left(\theta_{k}, \phi_{k}\right) S_{k}(n) e^{j 2 \pi f_{k} n_{0}}+\mathbf{n}_{m}^{x}\left(n+n_{0}\right),
\end{aligned}
$$

where $n_{0}$ is the constant sample delay.

We form the following matrices by using (6) and (7):

$$
\begin{aligned}
\mathbf{x}_{1}(n) & =\left[\mathbf{z}_{1}^{x}(n), \mathbf{z}_{2}^{x}(n), \ldots, \mathbf{z}_{M-1}^{x}(n)\right]^{T}=\mathbf{A} \mathbf{S}+\mathbf{N}_{1}, \\
\mathbf{y}_{1}(n) & =\left[\mathbf{z}_{2}^{x}(n), \mathbf{z}_{3}^{x}(n), \ldots, \mathbf{z}_{M}^{x}(n)\right]^{T}=\mathbf{A} \boldsymbol{\Phi}^{x} \mathbf{S}+\mathbf{N}_{2}, \\
\mathbf{x}_{2}(n) & =\left[\mathbf{z}_{1}^{y}(n), \mathbf{z}_{2}^{y}(n), \ldots, \mathbf{z}_{L-1}^{y}(n)\right]^{T}=\mathbf{B S}+\mathbf{N}_{3}, \\
\mathbf{y}_{2}(n) & =\left[\mathbf{z}_{2}^{y}(n), \mathbf{z}_{3}^{y}(n), \ldots, \mathbf{z}_{L}^{y}(n)\right]^{T}=\mathbf{B} \boldsymbol{\Phi}^{y} \mathbf{S}+\mathbf{N}_{4}, \\
\mathbf{y}_{3}(n) & =\left[\mathbf{z}_{1}^{x}\left(n+n_{0}\right), \mathbf{z}_{2}^{x}\left(n+n_{0}\right), \ldots, \mathbf{z}_{M-1}^{x}\left(n+n_{0}\right)\right]^{T} \\
& =\mathbf{A} \Phi^{t} \mathbf{S}+\mathbf{N}_{5},
\end{aligned}
$$

where

$$
\begin{aligned}
& \mathbf{S} \stackrel{\text { def }}{=}\left[\begin{array}{c}
S_{1}(n) \\
\vdots \\
S_{K}(n)
\end{array}\right], \quad \mathbf{N}_{1} \stackrel{\text { def }}{=}\left[\begin{array}{c}
\mathbf{n}_{1}^{x}(n) \\
\vdots \\
\mathbf{n}_{M-1}^{x}(n)
\end{array}\right], \\
& \mathbf{N}_{2} \stackrel{\text { def }}{=}\left[\begin{array}{c}
\mathbf{n}_{2}^{x}(n) \\
\vdots \\
\mathbf{n}_{M}^{x}(n)
\end{array}\right], \quad \mathbf{N}_{3} \stackrel{\text { def }}{=}\left[\begin{array}{c}
\mathbf{n}_{1}^{y}(n) \\
\vdots \\
\mathbf{n}_{L-1}^{y}(n)
\end{array}\right] \text {, } \\
& \mathbf{N}_{4} \stackrel{\text { def }}{=}\left[\begin{array}{c}
\mathbf{n}_{2}^{y}(n) \\
\vdots \\
\mathbf{n}_{L}^{y}(n)
\end{array}\right], \quad \mathbf{N}_{5} \stackrel{\text { def }}{=}\left[\begin{array}{c}
\mathbf{n}_{1}^{x}\left(n+n_{0}\right) \\
\vdots \\
\mathbf{n}_{M-1}^{x}\left(n+n_{0}\right)
\end{array}\right] \text {, } \\
& \mathbf{A}=\left[\mathbf{a}_{1}^{x}, \ldots, \mathbf{a}_{K}^{x}\right]=\left[\mathbf{q}^{x}\left(\theta_{1}, \phi_{1}\right) \otimes \mathbf{g}_{1}, \ldots, \mathbf{q}^{x}\left(\theta_{K}, \phi_{K}\right) \otimes \mathbf{g}_{K}\right], \\
& \mathbf{B}=\left[\mathbf{a}_{1}^{y}, \ldots, \mathbf{a}_{K}^{y}\right]=\left[\mathbf{q}^{y}\left(\theta_{1}, \phi_{1}\right) \otimes \mathbf{g}_{1}, \ldots, \mathbf{q}^{y}\left(\theta_{K}, \phi_{K}\right) \otimes \mathbf{g}_{K}\right], \\
& \begin{array}{c}
\mathbf{q}^{x}\left(\theta_{k}, \phi_{k}\right) \stackrel{\text { def }}{=}\left[\begin{array}{c}
1 \\
e^{j 2 \pi f_{k} F_{S} \Delta u_{k} / c} \\
\vdots \\
e^{j 2 \pi f_{k} F_{S}(M-2) \Delta u_{k} / c}
\end{array}\right], \\
\mathbf{q}^{y}\left(\theta_{k}, \phi_{k}\right) \stackrel{\text { def }}{=}\left[\begin{array}{c}
e^{j 2 \pi f_{k} F_{S} \Delta v_{k} / c} \\
\vdots \\
e^{j 2 \pi f_{k} F_{S}(L-2) \Delta v_{k} / c}
\end{array}\right] .
\end{array}
\end{aligned}
$$

$\mathbf{A}$ and $\mathbf{B}$ are the $6(M-1) \times K$ and $6(L-1) \times K$ matrices, respectively, and $\otimes$ denotes Kronecker product. $\Phi^{x}, \Phi^{y}$, and
$\Phi^{t}$ are diagonal $K \times K$ matrices and are given by

$$
\begin{aligned}
& \boldsymbol{\Phi}^{x}=\operatorname{diag}\left[\exp \frac{j 2 \pi f_{1} F_{S} \Delta u_{1}}{c}, \ldots, \exp \frac{j 2 \pi f_{K} F_{S} \Delta u_{K}}{c}\right], \\
& \boldsymbol{\Phi}^{y}=\operatorname{diag}\left[\exp \frac{j 2 \pi f_{1} F_{S} \Delta v_{1}}{c}, \ldots, \exp \frac{j 2 \pi f_{K} F_{S} \Delta v_{K}}{c}\right], \\
& \boldsymbol{\Phi}^{t}=\operatorname{diag}\left[\exp \left(j 2 \pi f_{1} n_{0}\right), \ldots, \exp \left(j 2 \pi f_{K} n_{0}\right)\right] .
\end{aligned}
$$

From $N$ snapshots, three data sets are formed as the following:

$$
\begin{gathered}
\mathbf{Z}_{1}=\left[\begin{array}{l}
\mathbf{X}_{1} \\
\mathbf{Y}_{1}
\end{array}\right]=\left[\begin{array}{lll}
\mathbf{x}_{1}(1) & \cdots & \mathbf{x}_{1}(N) \\
\mathbf{y}_{1}(1) & \cdots & \mathbf{y}_{1}(N)
\end{array}\right], \\
\mathbf{Z}_{2}=\left[\begin{array}{l}
\mathbf{X}_{2} \\
\mathbf{Y}_{2}
\end{array}\right]=\left[\begin{array}{lll}
\mathbf{x}_{2}(1) & \cdots & \mathbf{x}_{2}(N) \\
\mathbf{y}_{2}(1) & \cdots & \mathbf{y}_{2}(N)
\end{array}\right], \\
\mathbf{Z}_{3}=\left[\begin{array}{l}
\mathbf{X}_{1} \\
\mathbf{Y}_{3}
\end{array}\right]=\left[\begin{array}{lll}
\mathbf{x}_{1}(1) & \cdots & \mathbf{x}_{1}\left(N-n_{0}\right) \\
\mathbf{y}_{3}(1) & \cdots & \mathbf{y}_{3}\left(N-n_{0}\right)
\end{array}\right] .
\end{gathered}
$$

The key problem now is how to estimate the digital frequencies $\left\{f_{k}\right\}_{k=1}^{K}$ and arrival angles $\left\{\theta_{k}, \phi_{k}\right\}_{k=1}^{K}$ from the above data sets.

\section{ESPRIT-BASED FREQUENCY AND 2D ANGLE ESTIMATION ALGORITHM}

From (14), we have formed three distinct matrix-pencil pairs. This first matrix pencil $\mathbf{X}_{1}$ and $\mathbf{Y}_{1}$ has a spatial invariance along the $x$-axis and can yield estimates of the direction cosines $\left\{u_{k}, k=1, \ldots, K\right\}$. This second matrix pencil $\mathbf{X}_{2}$ and $\mathbf{Y}_{2}$ has a spatial invariance along the $y$-axis and can yield estimates of the direction cosines $\left\{v_{k}, k=1, \ldots, K\right\}$. This third matrix pencil $\mathbf{X}_{1}$ and $\mathbf{Y}_{3}$ has a temporal invariance and can yield estimates of the frequency $\left\{f_{k}, k=1, \ldots, K\right\}$.

The first step in ESPRIT is to compute the signalsubspace eigenvectors by eigendecomposing the data correlation matrices $\mathbf{R}_{1}=\mathbf{Z}_{1} \mathbf{Z}_{1}{ }^{H}, \mathbf{R}_{2}=\mathbf{Z}_{2} \mathbf{Z}_{2}{ }^{H}$, and $\mathbf{R}_{3}=\mathbf{Z}_{3} \mathbf{Z}_{3}{ }^{H}$ (where the superscript $H$ denotes the vector conjugate transpose operator). In the proposed algorithm, we basically modified the algorithm proposed in [18]. Thus, steps 2 to 6 are similar to and taken out from [18].

\section{(1) Deriving the frequency estimates}

Let $\mathbf{E}_{S}^{t}$ denote the $12(M-1) \times K$ signal-subspace eigenvector matrix whose $K$ columns are the $12(M-1) \times 1$ signalsubspace eigenvectors associated with the $K$ largest eigenvalues of $\mathbf{R}_{3}=\mathbf{Z}_{3} \mathbf{Z}_{3}{ }^{H}$.

The invariance structure of the matrix-pencil pair implies $\mathbf{E}_{S}^{t}$ can be decomposed into two $6(M-1) \times K$ subarrays such that [23]

$$
\mathbf{E}_{S}^{t}=\left[\begin{array}{c}
\mathbf{E}_{1}^{t} \\
\mathbf{E}_{2}^{t}
\end{array}\right]=\left[\begin{array}{c}
\mathbf{A} \mathbf{T}^{t} \\
\mathbf{A} \boldsymbol{\Phi}^{t} \mathbf{T}^{t}
\end{array}\right] .
$$

Because both $\mathbf{E}_{1}^{t}$ and $\mathbf{E}_{2}^{t}$ are full rank, a unique nonsingular $K \times K$ matrix $\Psi^{\mathbf{t}}$ exists such that [11]

$$
\begin{aligned}
\mathbf{E}_{1}^{t} \boldsymbol{\Psi}^{t} & =\mathbf{E}_{2}^{t} \Longrightarrow \mathrm{AT}^{t} \boldsymbol{\Psi}^{t}=\mathbf{A} \boldsymbol{\Phi}^{t} \mathbf{T}^{t} \\
& \Longrightarrow \boldsymbol{\Psi}^{t}=\left(\mathbf{T}^{t}\right)^{-1} \boldsymbol{\Phi}^{t} \mathbf{T}^{t} \Longrightarrow \boldsymbol{\Phi}^{t}=\mathbf{T}^{t} \boldsymbol{\Psi}^{t}\left(\mathbf{T}^{t}\right)^{-1}
\end{aligned}
$$


$\Psi^{t}$ can be estimated by the total-least-squares ESPRIT covariance algorithm (TLS-ESPRIT) [23].

$\Psi^{t}$ s eigenvalues equal $\left\{\left[\Phi^{t}\right]_{k k}=e^{j 2 \pi f_{k} n_{0}}, k=1, \ldots, K\right\}$,

$$
\exp \left(j 2 \pi \hat{f}_{k} n_{0}\right)=\left[\Phi^{t}\right]_{k k}
$$

If the maximum of the signal digital frequencies is $f_{\max }$, $n_{0}$ is chosen as the following:

$$
\left|2 \pi f_{\max } n_{0}\right| \leq \pi \Longrightarrow n_{0} \leq \frac{1}{2\left|f_{\max }\right|} .
$$

Then we can get the unambiguous frequency estimates:

$$
\hat{f}_{k}=\frac{\arg \left\{\left[\boldsymbol{\Phi}^{t}\right]_{k k}\right\}}{2 \pi n_{0}},
$$

where $\arg \{z\}$ is principle argument of the complex number $z$ between $-\pi$ and $\pi$.

\section{(2) Deriving the low-variance but ambiguous estimates of $u_{k}$}

Similarly, for the matrix pencil pair with spatial invariance along the $x$-axis, $\Psi^{x}$ s eigenvalues equal $\left\{\left[\Phi^{x}\right]_{k k}=\right.$ $\left.e^{j 2 \pi f_{k} F_{s} \Delta u_{k} / c}, k=1, \ldots, K\right\}$.

Because $\Delta \geq \lambda_{k}(k=1, \ldots, K)$ and $-1 \leq u_{k} \leq 1$, there exists a set of cyclically related candidates for the estimation of $u_{k}[18]$ :

$$
\begin{gathered}
\hat{u}_{k}\left(n_{u}\right)=\mu_{k}+\frac{n_{u} c}{\hat{f}_{k} F_{S} \Delta}, \\
\left.\left[\frac{\hat{f}_{k} F_{S} \Delta}{c}\left(-1-\mu_{k}\right)\right] \leq n_{u} \leq \mid \frac{\hat{f}_{k} F_{S} \Delta}{c}\left(-1-\mu_{k}\right)\right\rfloor, \\
\mu_{k}=\frac{\arg \left\{\left[\Phi^{x}\right]_{k k}\right\} \cdot c}{2 \pi \hat{f}_{k} F_{S} \Delta},
\end{gathered}
$$

where $\lceil x\rceil$ is the smallest integer not less than $x ;\lfloor x\rfloor$ is the largest integer not greater than $x$.

\section{(3) Deriving the low-variance but ambiguous estimates of $v_{k}$}

Similarly, for the matrix pencil pair with spatial invariance along the $y$-axis, $\Psi^{y}$ 's eigenvalues equal $\left\{\left[\boldsymbol{\Phi}^{y}\right]_{k k}=\right.$ $\left.e^{j 2 \pi f_{k} F_{s} \Delta v_{k} / c}, k=1, \ldots, K\right\}$.

There exists a set of cyclically related candidates for the estimation of $v_{k}[18]$ :

$$
\begin{gathered}
\hat{v}_{k}\left(n_{v}\right)=v_{k}+\frac{n_{v} c}{\hat{f}_{k} F_{S} \Delta}, \\
{\left[\frac{\hat{f}_{k} F_{S} \Delta}{c}\left(-1-v_{k}\right)\right] \leq n_{v} \leq\left\lfloor\frac{\hat{f}_{k} F_{S} \Delta}{c}\left(1-v_{k}\right)\right],} \\
v_{k}=\frac{\arg \left\{\left[\Phi^{y}\right]_{k k}\right\} \cdot c}{2 \pi \hat{f}_{k} F_{S} \Delta} .
\end{gathered}
$$

\section{(4) Deriving the unambiguous coarse reference estimates of $u_{k}$ and $v_{k}$ from ESPRIT's eigenvector}

$\Psi^{x}$ 's right eigenvectors constitute the columns of $\mathbf{T}^{x}$. From [11], we have the following:

$$
\widehat{\mathbf{A}}=0.5\left\{\mathbf{E}_{1}^{x}\left(\mathbf{T}^{x}\right)^{-1}+\mathbf{E}_{2}^{x}\left(\mathbf{T}^{x}\right)^{-1}\left(\boldsymbol{\Phi}^{x}\right)^{-1}\right\}
$$

With noise, the above estimation becomes only approximate.

We have the array manifold estimates from (10):

$$
\begin{aligned}
\hat{\mathbf{a}}_{k}^{x} & =\hat{\mathbf{q}}^{x}\left(\theta_{k}, \phi_{k}\right) \otimes \hat{\mathbf{g}}_{k}=\hat{\mathbf{q}}^{x}\left(\theta_{k}, \phi_{k}\right) \otimes\left[\begin{array}{c}
\hat{\mathbf{e}}_{k} \\
\hat{\mathbf{h}}_{k}
\end{array}\right] \\
& =\left[\begin{array}{c}
\hat{q}_{1}^{x}\left(\theta_{k}, \phi_{k}\right) \hat{\mathbf{e}}_{k} \\
\hat{q}_{1}^{x}\left(\theta_{k}, \phi_{k}\right) \hat{\mathbf{h}}_{k} \\
\vdots \\
\hat{q}_{M-1}^{x}\left(\theta_{k}, \phi_{k}\right) \hat{\mathbf{e}}_{k} \\
\hat{q}_{M-1}^{x}\left(\theta_{k}, \phi_{k}\right) \hat{\mathbf{h}}_{k}
\end{array}\right] .
\end{aligned}
$$

Define

$$
\mathbf{b}_{i}(k)=\hat{q}_{i}^{x}\left(\theta_{k}, \phi_{k}\right) \widehat{\mathbf{e}}_{k}, \quad \mathbf{c}_{i}(k)=\hat{q}_{i}^{x}\left(\theta_{k}, \phi_{k}\right) \hat{\mathbf{h}}_{k}
$$

$i=1,2, \ldots, M-1$.

Note that

$$
\begin{aligned}
\frac{\mathbf{b}_{i}(k)}{\left\|\mathbf{b}_{i}(k)\right\|} \times \frac{\mathbf{c}_{i}^{*}(k)}{\left\|\mathbf{c}_{i}(k)\right\|} & =\frac{q_{i}^{x}\left(\theta_{k}, \phi_{k}\right) \hat{\mathbf{e}}_{k}}{\left\|q_{i}^{x}\left(\theta_{k}, \phi_{k}\right) \hat{\mathbf{e}}_{k}\right\|} \times \frac{q_{i}^{x^{*}}\left(\theta_{k}, \phi_{k}\right) \hat{\mathbf{h}}_{k}^{*}}{\left\|q_{i}^{x}\left(\theta_{k}, \phi_{k}\right) \hat{\mathbf{h}}_{k}\right\|} \\
& =\frac{\hat{\mathbf{e}}_{k}}{\left\|\hat{\mathbf{e}}_{k}\right\|} \frac{\hat{\mathbf{h}}_{k}^{*}}{\left\|\hat{\mathbf{h}}_{k}\right\|}
\end{aligned}
$$

So we can get the estimate of Ponyting vector:

$$
\hat{\mathbf{p}}_{k}^{x}=\frac{1}{M-1} \sum_{i=1}^{M-1} \frac{\mathbf{b}_{i}(k)}{\left\|\mathbf{b}_{i}(k)\right\|} \times \frac{\mathbf{c}_{i}^{*}(k)}{\left\|\mathbf{c}_{i}(k)\right\|}
$$

Unambiguous but high-variance estimates $\left\{\hat{p}_{x k}^{x}, \hat{p}_{y k}^{x}, \hat{p}_{z k}^{x}\right\}$ for $\left\{u_{k}, v_{k}, w_{k}\right\}$ have been achieved. This is the so-called vector cross-product estimator who is pioneered by Nehorai and Paldi $[1,2]$ and firstly adapted to ESPRIT by Wong and Zoltowski $[11,24]$.

Similarly, for the matrix pencil with spatial invariance along the $y$-axis, we can get another set of unambiguous but high-variance estimates $\hat{\mathbf{p}}_{k}^{y}$ for $\left\{u_{k}, v_{k}, w_{k}\right\}$. For the matrix pencil with temporal invariance, we can get $\hat{\mathbf{p}}_{k}^{t}$.

\section{(5) Pairing the direction-cosine estimates and frequency estimates}

The orderings of $\left\{\hat{p}_{x i}^{t}, \hat{p}_{y i}^{t}, \hat{p}_{z i}^{t}, i=1,2, \ldots, K\right\},\left\{\hat{p}_{x j}^{x}, \hat{p}_{y j}^{x}, \hat{p}_{z j}^{x}\right.$, $j=1,2, \ldots, K\}$ and $\left\{\hat{p}_{x k}^{y}, \hat{p}_{y k}^{y}, \hat{p}_{z k}^{y}, k=1,2, \ldots, K\right\}$ are different and need to be paired. $\left\{\hat{p}_{x i}^{t}, \hat{p}_{y i}^{t}, \hat{p}_{z i}^{t}\right\}$ can be easily paired 
with $\left\{\hat{p}_{x j}^{x}, \hat{p}_{y j}^{x}, \hat{p}_{z j}^{x}\right\}$ and $\left\{\hat{p}_{x k}^{y}, \hat{p}_{y k}^{y}, \hat{p}_{z k}^{y}\right\}$ as follows [18]:

$$
\begin{aligned}
\left\{j_{1}^{0}, \ldots, j_{K}^{0}\right\} & =\arg \min \left\|\left[\hat{\mathbf{p}}_{1}^{t}, \ldots, \hat{\mathbf{p}}_{K}^{t}\right]-\left[\hat{\mathbf{p}}_{j_{1}}^{x}, \ldots, \hat{\mathbf{p}}_{j_{K}}^{x}\right]\right\|, \\
\left\{k_{1}^{0}, \ldots, k_{K}^{0}\right\} & =\arg \min \left\|\left[\hat{\mathbf{p}}_{1}^{t}, \ldots, \hat{\mathbf{p}}_{K}^{t}\right]-\left[\hat{\mathbf{p}}_{k_{1}}^{y}, \ldots, \hat{\mathbf{p}}_{k_{K}}^{y}\right]\right\| .
\end{aligned}
$$

The above minimization is with respect to all possible permutations of $\left\{k_{1}, \ldots, k_{K}\right\}$ and $\left\{j_{1}, \ldots, j_{K}\right\}$.

From $\hat{\mathbf{p}}_{k}^{t}, \hat{\mathbf{p}}_{k}^{x}, \hat{\mathbf{p}}_{k}^{y}$ we may form a $\hat{\mathbf{p}}_{k}$ :

$$
\hat{\mathbf{p}}_{k}=\left[\begin{array}{c}
\hat{p}_{x k} \\
\hat{p}_{y k} \\
\hat{p}_{z k}
\end{array}\right]=\frac{\hat{\mathbf{p}}_{k}^{t}+\hat{\mathbf{p}}_{k}^{x}+\hat{\mathbf{p}}_{k}^{y}}{3} .
$$

$\left\{\hat{p}_{x i}^{t}, \hat{p}_{y i}^{t}, \hat{p}_{z i}^{t}\right\}$ are already paired with $f_{i}$, and $\left\{\hat{p}_{x j}^{x}, \hat{p}_{y j}^{x}\right.$, $\left.\hat{p}_{z j}^{x}\right\}$ with $\mu_{j},\left\{\hat{p}_{x k}^{y}, \hat{p}_{y k}^{y}, \hat{p}_{z k}^{y}\right\}$ with $v_{k}$. It follows that $\left\{\hat{f}_{1}, \ldots\right.$, $\left.\hat{f}_{K}\right\}$ is to be paired with $\left\{\hat{\mu}_{j_{1}^{0}}, \ldots, \hat{\mu}_{j_{K}^{0}}\right\}$ and $\left\{\hat{v}_{k_{1}^{0}}, \ldots, \hat{v}_{k_{K}^{0}}\right\}[18]$.

\section{(6) Disambiguation of the low-variance estimates of direction-cosine from ESPRIT's eigenvalues [18]}

The disambiguated estimates are

$$
\hat{u}_{k}\left(n_{u}\right)=\mu_{k}+\frac{n_{u}^{\circ} c}{\hat{f}_{k} F_{S} \Delta}, \quad \hat{v}_{k}\left(n_{v}\right)=v_{k}+\frac{n_{v}^{\circ} c}{\hat{f}_{k} F_{S} \Delta},
$$

where $n_{u}^{\circ}$ and $n_{v}^{\circ}$ may be separately estimated as

$$
\begin{aligned}
& n_{u}^{\circ}=\underset{n_{u}}{\arg \min }\left|\hat{p}_{x k}-\mu_{k}-\frac{n_{u} c}{\hat{f}_{k} F_{S} \Delta}\right|, \\
& n_{v}^{\circ}=\underset{n_{v}}{\arg \min }\left|\hat{p}_{y k}-v_{k}-\frac{n_{v} c}{\hat{f}_{k} F_{S} \Delta}\right| .
\end{aligned}
$$

\section{(7) The 2D arrival angle estimation}

We can calculate low-variance 2D arrival angle estimates from direction-cosine estimates out of ESPRIT's eigenvalues

$$
\begin{gathered}
\hat{\theta}_{k}=\arcsin \left(\sqrt{\hat{u}_{k}^{2}+\hat{v}_{k}^{2}}\right), \\
\hat{\phi}_{k}=\arctan \left(\frac{\hat{v}_{k}}{\hat{u}_{k}}\right) .
\end{gathered}
$$

Similarly, we can calculate the high-variance 2D arrival angle estimates from direction-cosine estimates out of ESPRIT's eigenvectors

$$
\begin{gathered}
\hat{\theta}_{k}=\arcsin \left(\sqrt{\hat{p}_{x k}^{2}+\hat{p}_{y k}^{2}}\right), \\
\hat{\phi}_{k}=\arctan \left(\frac{\hat{p}_{y k}}{\hat{p}_{x k}}\right) .
\end{gathered}
$$

Note that $\hat{p}_{z k}$ may be applied to judge the quadrant of $\hat{\theta}_{k}$.

\section{SIMULATIONS}

Several simulations are presented to verify the effectiveness of the proposed ESPRIT-based frequency and 2D angle estimation algorithm. In these simulations, the total-least-squares

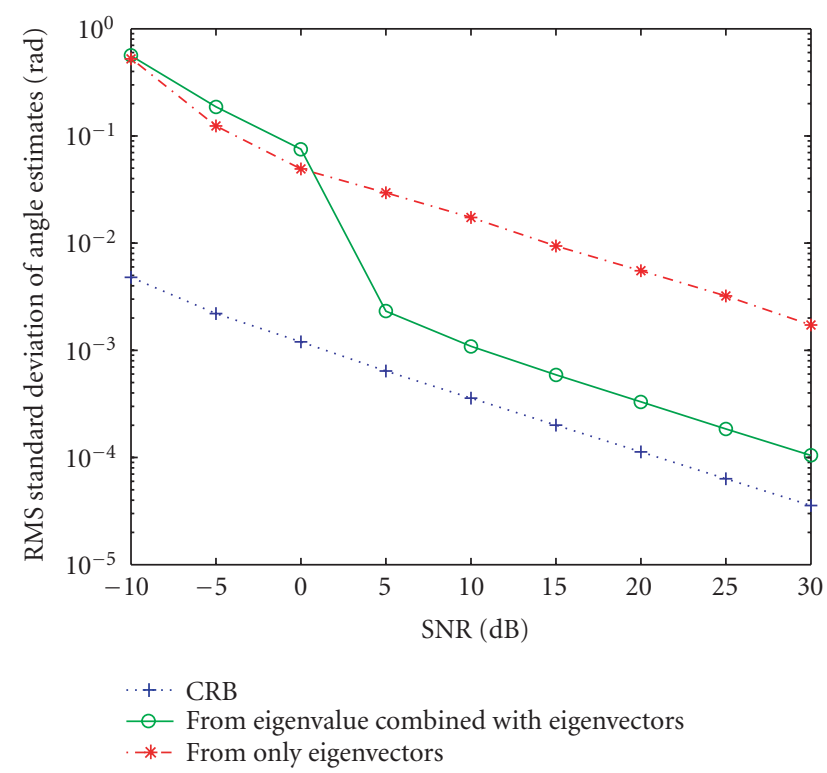

FIGURE 1: The RMS standard deviation of $\left(\hat{\theta}_{k}, \hat{\phi}_{k}, k=1,2\right)$ versus SNR: the two uncorrelated sources $\left\{\theta_{1}, \theta_{2}\right\}=\left(30^{\circ}, 60^{\circ}\right),\left\{\phi_{1}, \phi_{2}\right\}=$ $\left(40^{\circ},-60^{\circ}\right),\left\{\gamma_{1}, \gamma_{2}\right\}=\left(0^{\circ}, 45^{\circ}\right),\left\{\eta_{1}, \eta_{2}\right\}=\left(0^{\circ}, 90^{\circ}\right),\left\{f_{1}, f_{2}\right\}=$ $(0.3,0.4)$ impinge upon an $L$-shaped vector sensor, 100 snapshots per experiment, 300 experiments per data point.

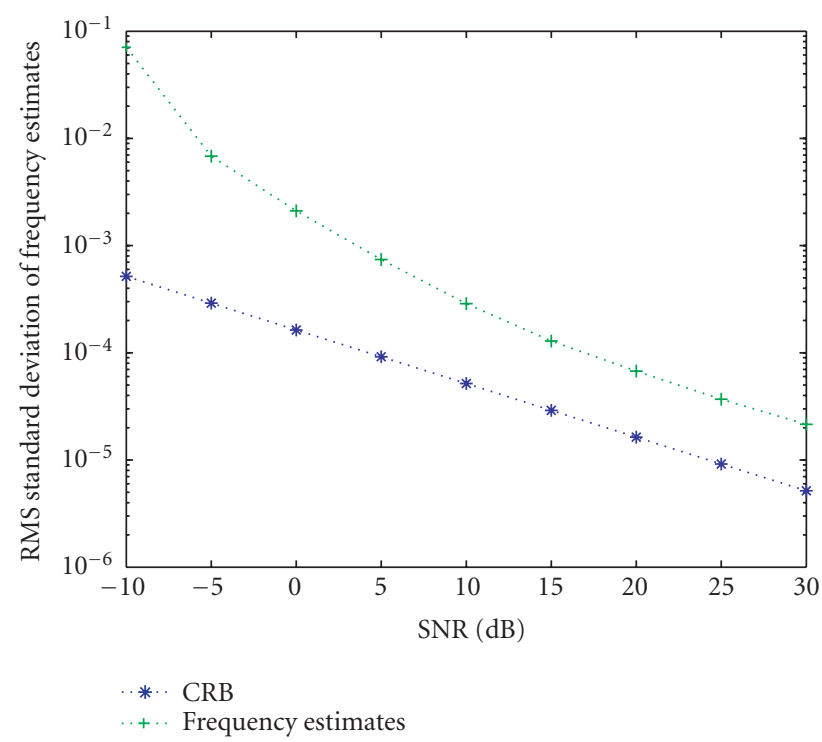

FIGURE 2: The RMS standard deviation of $\left(\hat{f}_{k}, k=1,2\right)$ versus SNR, same settings as an Figure 1.

ESPRIT covariance algorithm (TLS-ESPRIT) [23] is used. We consider the scenario of the two signals impinging one uniform $L$-shaped array and $M=4, L=4$. All the signal source's energy $\mathbf{P}$ is unity and $n_{0}=1$. The intersensor spacing is chosen as $\Delta=10 * \lambda_{\min } / 2\left(\lambda_{\min }=c /\left(f_{\max } F_{s}\right)\right)$ except for the example in Figures 4 and 5.

Figures 1 and 2 give the RMS standard deviations of $\left(\hat{\theta}_{k}\right.$, $\left.\hat{\phi}_{k}, k=1,2\right)$ and $\left(\hat{f}_{k}, k=1,2\right)$ versus SNR, respectively. The 


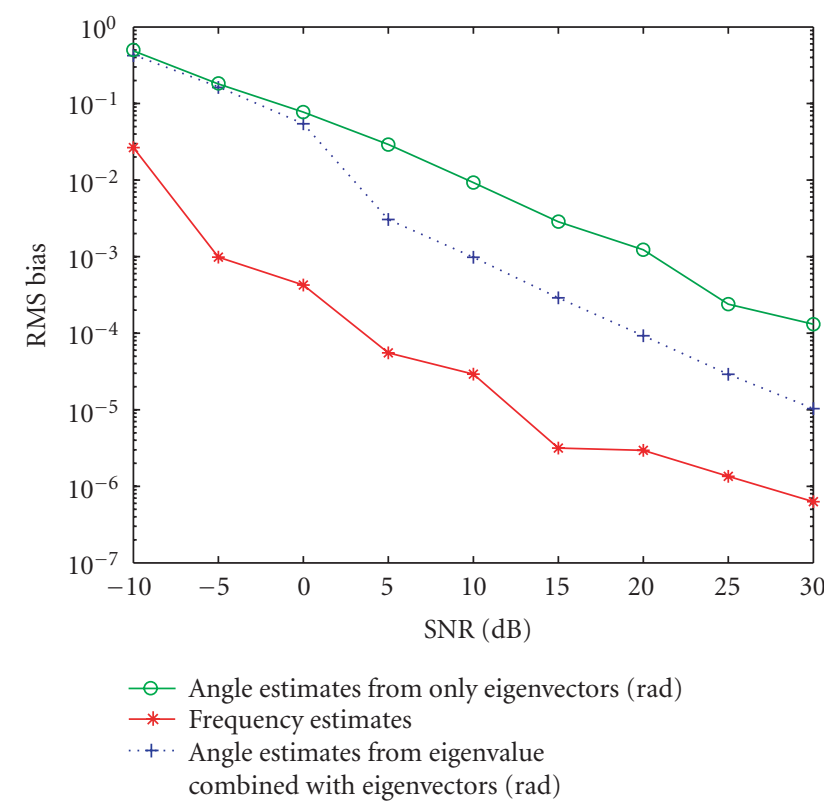

FIgURE 3: The RMS bias of $\left(\hat{\theta}_{k}, \hat{\phi}_{k}, k=1,2\right)$ and $\left(\hat{f}_{k}, k=1,2\right)$ versus SNR, same settings as in Figure 1.

parameters of the two signals are $\left\{\theta_{1}, \theta_{2}\right\}=\left(30^{\circ}, 60^{\circ}\right),\left\{\phi_{1}\right.$, $\left.\phi_{2}\right\}=\left(40^{\circ},-60^{\circ}\right),\left\{\gamma_{1}, \gamma_{2}\right\}=\left(0^{\circ}, 45^{\circ}\right),\left\{\eta_{1}, \eta_{2}\right\}=\left(0^{\circ}, 90^{\circ}\right)$, $\left\{f_{1}, f_{2}\right\}=(0.3,0.4)$. Figure 3 gives the corresponding RMS bias versus SNR. The proposed algorithm successfully resolves all the two electromagnetic source parameters including frequency and 2D angles. Figures 1 and 3 show that the angle estimates from ESPRIT's eigenvalues combined with eigenvectors have better performance than angle estimates obtained from only ESPRIT's eigenvectors at SNR's above $1 \mathrm{~dB}$. It is observed that the RMS bias of angle estimates is less than $0.2^{\circ}$ at SNR's above $5 \mathrm{~dB}$ and $0.1^{\circ}$ at SNR's above $10 \mathrm{~dB}$. RMS standard deviation of frequency estimates is less than one order of magnitude greater than the CRB at SNR's above $0 \mathrm{~dB}$.

Figures 4 and 5, respectively, give the RMS standard deviations and bias of $\left(\hat{\theta}_{k}, \hat{\phi}_{k}, k=1,2\right)$ versus intersensor spacing when SNR $=15$. The parameters of the two signals are $\left\{\theta_{1}, \theta_{2}\right\}=\left(60^{\circ}, 30^{\circ}\right),\left\{\phi_{1}, \phi_{2}\right\}=\left(40^{\circ},-60^{\circ}\right)$, $\left\{\gamma_{1}, \gamma_{2}\right\}=\left(0^{\circ}, 45^{\circ}\right),\left\{\eta_{1}, \eta_{2}\right\}=\left(0^{\circ}, 90^{\circ}\right),\left\{f_{1}, f_{2}\right\}=(0.4,0.5)$. Figure 3 shows that the standard deviations and bias of angle estimates from the eigenvalues combined with eigenvectors decrease as the intersensor spacing increases when $\Delta<$ $60 \lambda_{\min } / 2$. But the performance of angle estimates obtained from only the eigenvectors remains relatively constant as the inter-sensor spacing increases. Note that when $\Delta \geq 60 \lambda_{\min } / 2$, the standard deviations and bias of angle estimates from the eigenvalues combined with eigenvectors begin to increase as the intersensor spacing increases. In fact, this phenomenon has been explained in [18].

From (29), it can be seen that the performance of frequency estimation may affect the performance of low-variance angle estimation. Figure 6 gives the RMS standard deviation of $\left(\hat{\theta}_{k}, \hat{\phi}_{k}, k=1,2\right)$ versus SNR. The signal parameters

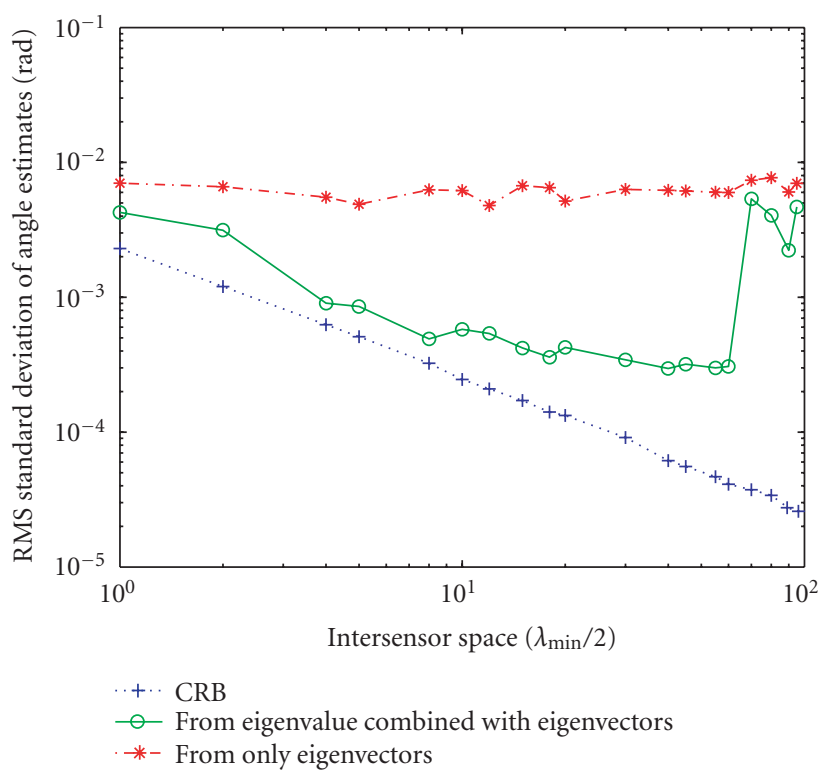

FIGURE 4: The RMS standard deviation of $\left(\hat{\theta}_{k}, \hat{\phi}_{k}, k=1,2\right)$ versus intersensor spacing when SNR $=15 \mathrm{~dB}$ : the two uncorrelated sources $\left\{\theta_{1}, \theta_{2}\right\}=\left(60^{\circ}, 30^{\circ}\right),\left\{\phi_{1}, \phi_{2}\right\}=\left(40^{\circ},-60^{\circ}\right),\left\{\gamma_{1}, \gamma_{2}\right\}=$ $\left(0^{\circ}, 45^{\circ}\right),\left\{\eta_{1}, \eta_{2}\right\}=\left(0^{\circ}, 90^{\circ}\right),\left\{f_{1}, f_{2}\right\}=(0.4,0.5)$ impinge upon an $L$-shaped vector sensor, 100 snapshots per experiment, 300 experiments per data point.

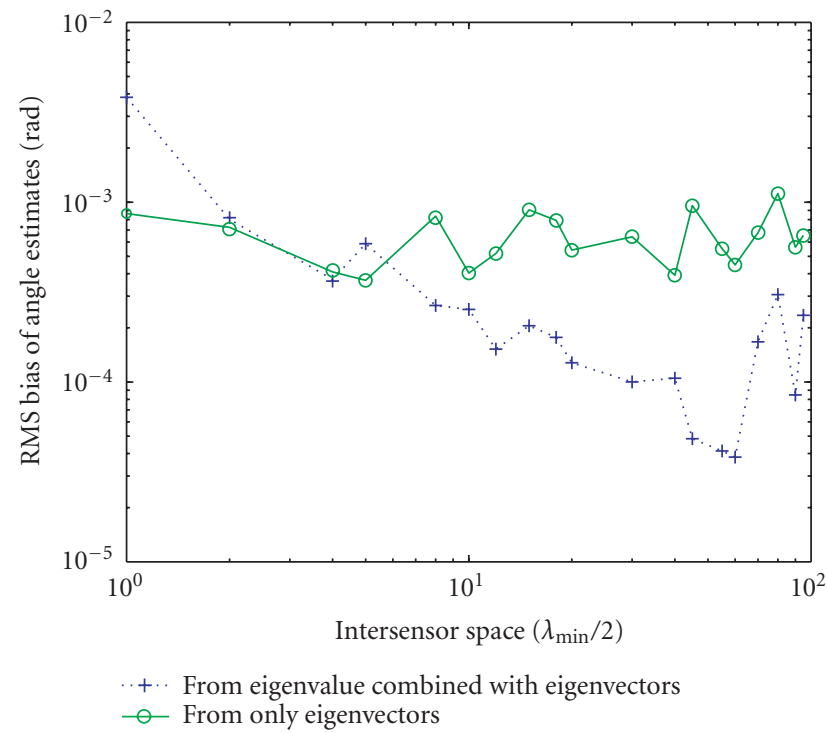

FIGURE 5: The RMS bias of $\left(\hat{\theta}_{k}, \hat{\phi}_{k}, k=1,2\right)$ versus intersensor spacing when $\mathrm{SNR}=15 \mathrm{~dB}$, same settings as in Figure 4 .

are the same as in Figure 1 except that $\left\{f_{1}, f_{2}\right\}=(0.35,0.4)$. One curve is calculated from the low-variance angle estimation algorithm when the signal frequencies are not known and estimated. Another curve is calculated by the low-variance angle estimation algorithm when the signal frequencies are known. It is shown that when signal frequencies are known, the RMS standard deviation of angle estimates is 


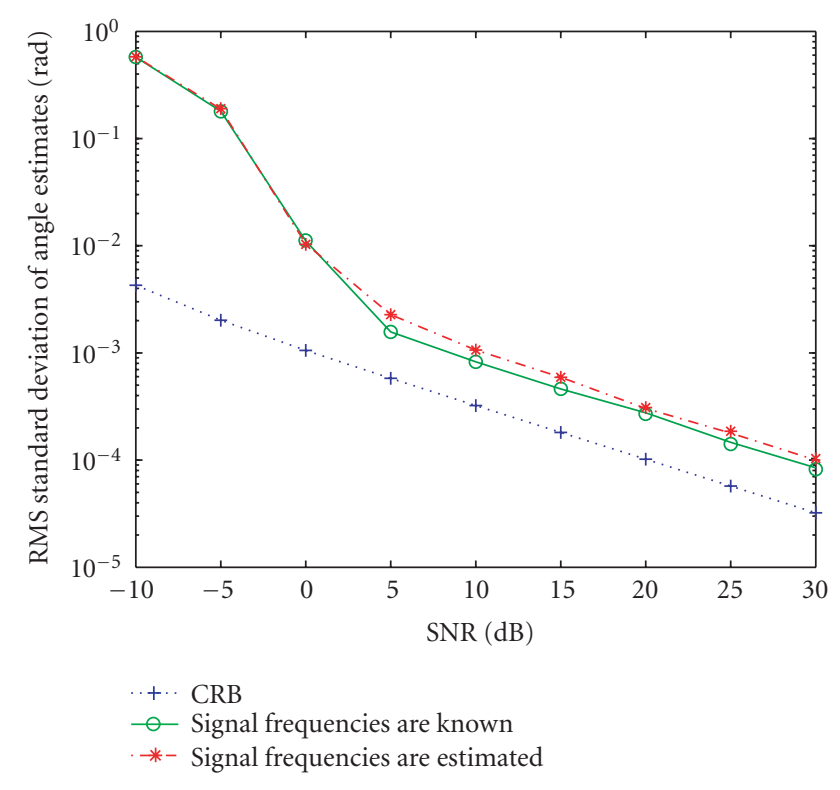

FIgURE 6: The RMS standard deviations of $\left(\hat{\theta}_{k}, \hat{\phi}_{k}, k=1,2\right)$ versus SNR from low-variance angle estimation, same settings as in Figure 1 except that $\left\{f_{1}, f_{2}\right\}=(0.35,0.4)$.

just slightly lower than that when signal frequencies are estimated. Our simulations also show that RMS bias of the lowvariance angle estimates when frequencies are known is almost the same as that when frequencies are estimated.

Figure 7 gives the RMS standard deviation of $\left(\hat{\theta}_{k}, \hat{\phi}_{k}, k=\right.$ $1,2)$ versus elevation angle of the first signal when SNR = $15 \mathrm{~dB}$. The parameters of the two signals are $\theta_{2}=45^{\circ},\left\{\phi_{1}\right.$, $\left.\phi_{2}\right\}=\left(25^{\circ},-30^{\circ}\right),\left\{\gamma_{1}, \gamma_{2}\right\}=\left(0^{\circ}, 45^{\circ}\right),\left\{\eta_{1}, \eta_{2}\right\}=\left(0^{\circ}, 90^{\circ}\right)$, $\left\{f_{1}, f_{2}\right\}=(0.3,0.4)$. It is observed that the standard deviations of angle estimates from the eigenvalues combined with eigenvectors are greater than angle estimates from ESPRIT eigenvectors when elevation angle nears $90^{\circ}$.

Figure 8 gives the RMS standard deviation of $\left(\hat{\theta}_{k}, \hat{\phi}_{k}, k=\right.$ $1,2)$ versus azimuth angle of the first signal when SNR = $15 \mathrm{~dB}$. The signal parameters are the same as in Figure $7 \mathrm{ex}-$ cept that $\left\{\theta_{1}, \theta_{2}\right\}=\left(30^{\circ}, 45^{\circ}\right), \phi_{2}=-30^{\circ}$. It is shown that the RMS standard deviation of angle estimates from two estimation methods almost does not change as the azimuth angle of the first signal is changed.

Figure 9 gives the RMS standard deviation of $\left(\hat{\theta}_{k}, \hat{\phi}_{k}, k=\right.$ $1,2)$ and $\left(\hat{f}_{k}, k=1,2\right)$ versus the number of snapshots when SNR $=15 \mathrm{~dB}$. The parameters of the two signals are $\left\{\theta_{1}, \theta_{2}\right\}=\left(60^{\circ}, 30^{\circ}\right),\left\{\phi_{1}, \phi_{2}\right\}=\left(40^{\circ},-60^{\circ}\right),\left\{\gamma_{1}, \gamma_{2}\right\}=$ $\left(0^{\circ}, 45^{\circ}\right),\left\{\eta_{1}, \eta_{2}\right\}=\left(0^{\circ}, 90^{\circ}\right),\left\{f_{1}, f_{2}\right\}=(0.3,0.4)$. It is shown that the RMS standard deviation decreases slowly as the number of snapshots increases for the number of snapshots exceeding 50 .

Figure 10 gives the RMS standard deviation and bias of $\left(\hat{f}_{k}, k=1,2\right)$ versus the difference $\Delta f$ of two signal frequencies when SNR $=15 \mathrm{~dB}$. The signal parameters are the same as in Figure 9 except that $\left\{f_{1}, f_{2}\right\}=(0.4-\Delta f, 0.4)$.

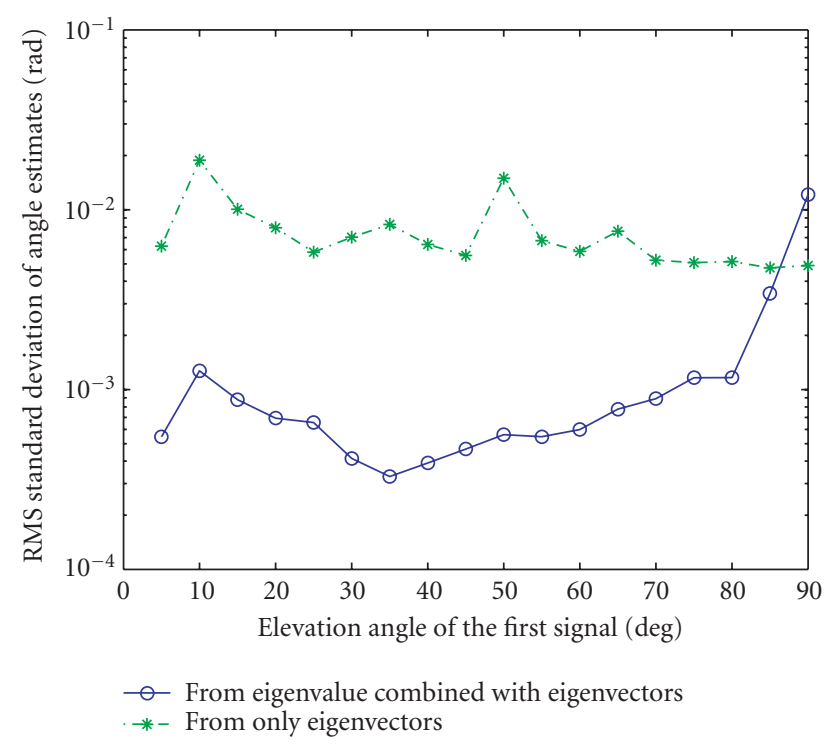

FIGURE 7: The RMS standard deviations of $\left(\hat{\theta}_{k}, \hat{\phi}_{k}, k=1,2\right)$ versus elevation angle of the first signal when $\mathrm{SNR}=15 \mathrm{~dB}$. The parameters of the two signals are $\theta_{2}=45^{\circ},\left\{\phi_{1}, \phi_{2}\right\}=\left(25^{\circ},-30^{\circ}\right)$, $\left\{\gamma_{1}, \gamma_{2}\right\}=\left(0^{\circ}, 45^{\circ}\right),\left\{\eta_{1}, \eta_{2}\right\}=\left(0^{\circ}, 90^{\circ}\right),\left\{f_{1}, f_{2}\right\}=(0.3,0.4), 100$ snapshots per experiment, 300 experiments per data point.

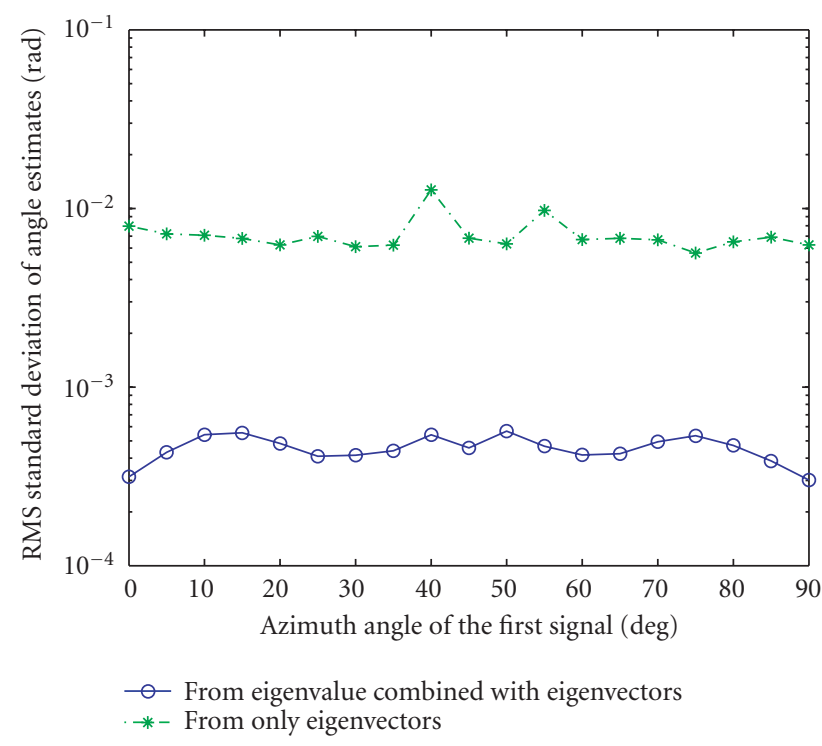

FIGURE 8: The RMS standard deviation of $\left(\hat{\theta}_{k}, \hat{\phi}_{k}, k=1,2\right)$ versus azimuth angle of the first signal when $\mathrm{SNR}=15 \mathrm{~dB}$, same setting as in Figure 7 except that $\left\{\theta_{1}, \theta_{2}\right\}=\left(30^{\circ}, 45^{\circ}\right)$ and $\phi_{2}=-30^{\circ}$.

It is observed that when $\Delta f$ is 0.004 , the RMS bias is about $2.5 \mathrm{e}-4$ and standard deviation is about $1.6 \mathrm{e}-3$, which shows that two signal frequencies can be separated. Note that just 50 snapshots are used here. For discrete Fourier transform when 50 snapshots are used, the frequency discrimination is just $1 / 50=0.02$. 


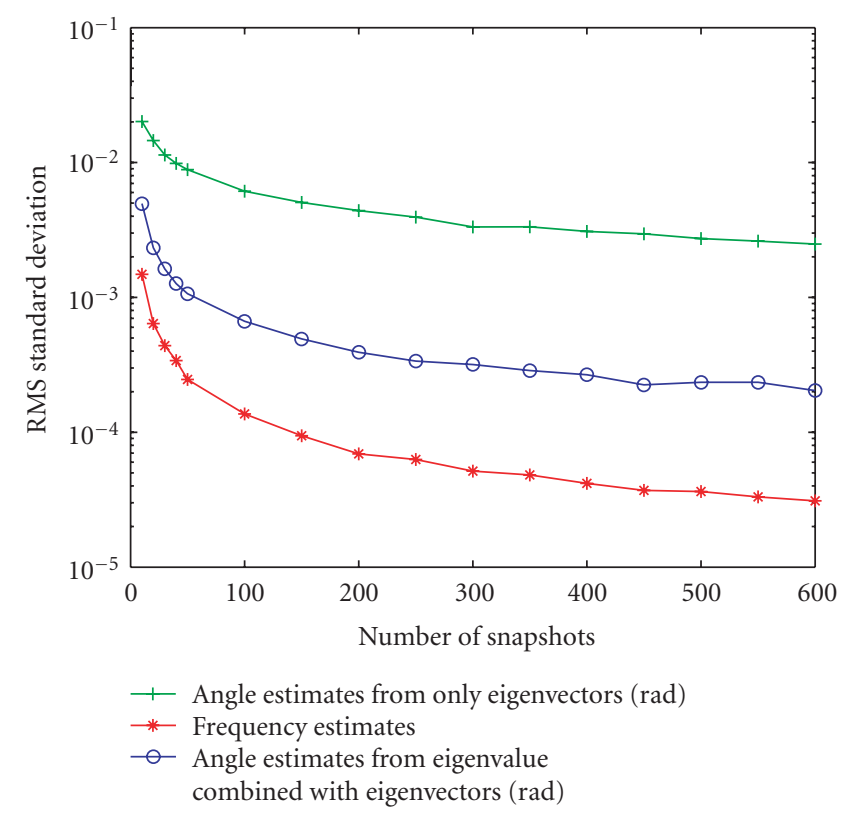

FIGURE 9: The RMS standard deviation of $\left(\hat{\theta}_{k}, \hat{\phi}_{k}, k=1,2\right)$ and $\left(\hat{f}_{k}, k=1,2\right)$ versus the number of snapshots when SNR = $15 \mathrm{~dB}$. The parameters of the two signals are $\left\{\theta_{1}, \theta_{2}\right\}=\left(60^{\circ}, 30^{\circ}\right)$, $\left\{\phi_{1}, \phi_{2}\right\}=\left(40^{\circ},-60^{\circ}\right),\left\{\gamma_{1}, \gamma_{2}\right\}=\left(0^{\circ}, 45^{\circ}\right),\left\{\eta_{1}, \eta_{2}\right\}=\left(0^{\circ}, 90^{\circ}\right)$, $\left\{f_{1}, f_{2}\right\}=(0.3,0.4), 100$ snapshots per experiment, 300 experiments per data point.

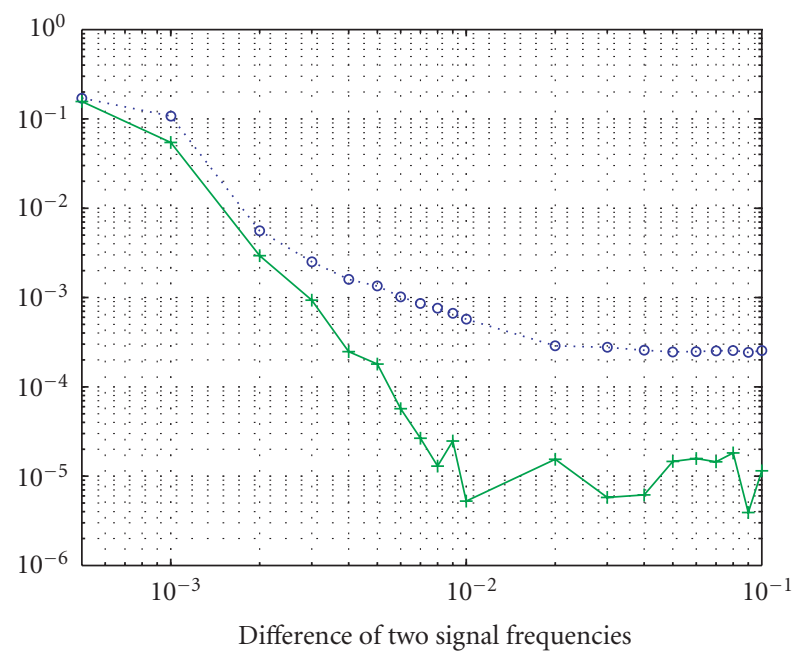

๑.. RMS standard deviation

1 RMS bias

FIgURE 10: The RMS standard deviations and bias of $\left(\hat{f}_{k}, k=1,2\right)$ versus the difference $\Delta f$ of two signal frequencies when SNR $=$ $15 \mathrm{~dB}$, same settings as in Figure 9 except that $\left\{f_{1}, f_{2}\right\}=(0.4-$ $\Delta f, 0.4), 50$ snapshots per experiment, 300 experiments per data point.

\section{CONCLUSION}

In this paper, we propose an ESPRIT-based algorithm that yields 2D angle and frequency estimates. This algorithm can achieve extended-aperture arrival angle estimation even though using a sparse electromagnetic vector sensor array. Good frequency discrimination obtained even though there are little samples used. Although we only consider the $L$ shaped array here, the approach may be implemented using a variety of array geometries.

\section{ACKNOWLEDGMENTS}

This work is supported by City University of Hong Kong Strategic Grant 7001697. This work is done when Fei Ji was visiting City University of Hong Kong.

\section{REFERENCES}

[1] A. Nehorai and E. Paldi, "Vector sensor processing for electromagnetic source localization," in Proceedings of the 25th Asilomar Conference on Signals, Systems and Computers, vol. 1, pp. 566-572, Pacific Grove, Calif, USA, November 1991.

[2] A. Nehorai and E. Paldi, "Vector-sensor array processing for electromagnetic source localization," IEEE Transactions on Signal Processing, vol. 42, no. 2, pp. 376-398, 1994.

[3] J. Li, "Direction and polarization estimation using arrays with small loops and short dipoles," IEEE Transactions on Antennas and Propagation, vol. 41, no. 3, pp. 379-386, 1993.

[4] B. Hochwald and A. Nehorai, "Identifiability in array processing models with vector-sensor applications," IEEE Transactions on Signal Processing, vol. 44, no. 1, pp. 83-95, 1996.

[5] K.-C. Ho, K.-C. Tan, and W. Ser, "Investigation on number of signals whose directions-of-arrival are uniquely determinable with an electromagnetic vector sensor," Signal Processing, vol. 47, no. 1, pp. 41-54, 1995.

[6] K.-C. Tan, K.-C. Ho, and A. Nehorai, "Uniqueness study of measurements obtainable with arrays of electromagnetic vector sensors," IEEE Transactions on Signal Processing, vol. 44, no. 4, pp. 1036-1039, 1996.

[7] B. Hochwald and A. Nehorai, "Polarimetric modeling and parameter estimation with applications to remote sensing," IEEE Transactions on Signal Processing, vol. 43, no. 8, pp. 1923-1935, 1995.

[8] K.-C. Ho, K.-C. Tan, and B. T. G. Tan, "Efficient method for estimating directions-of-arrival of partially polarized signals with electromagnetic vector sensors," IEEE Transactions on Signal Processing, vol. 45, no. 10, pp. 2485-2498, 1997.

[9] K.-C. Ho, K.-C. Tan, and A. Nehorai, "Estimating directions of arrival of completely and incompletely polarized signals with electromagnetic vector sensors," IEEE Transactions on Signal Processing, vol. 47, no. 10, pp. 2845-2852, 1999.

[10] K. T. Wong, "Direction finding/polarization estimationdipole and/or loop triads," IEEE Transactions on Aerospace and Electronic Systems, vol. 37, no. 2, pp. 679-684, 2001.

[11] K. T. Wong and M. D. Zoltowski, "Uni-vector-sensor ESPRIT for multisource azimuth, elevation, and polarization estimation," IEEE Transactions on Antennas and Propagation, vol. 45, no. 10, pp. 1467-1474, 1997.

[12] A. Nehorai and P. Tichavsky, "Cross-product algorithms for source tracking using an EM vector sensor," IEEE Transactions on Signal Processing, vol. 47, no. 10, pp. 2863-2867, 1999.

[13] C. C. Ko, J. Zhang, and A. Nehorai, "Separation and tracking of multiple broadband sources with one electromagnetic vector sensor," IEEE Transactions on Aerospace and Electronic Systems, vol. 38, no. 3, pp. 1109-1116, 2002. 
[14] K. T. Wong, "Blind beamforming geolocation for widebandFFHs with unknown hop-sequences," IEEE Transactions on Aerospace and Electronic Systems, vol. 37, no. 1, pp. 65-76, 2001.

[15] D. Rahamim, J. Tabrikian, and R. Shavit, "Source localization using vector sensor array in a multipath environment," IEEE Transactions on Signal Processing, vol. 52, no. 11, pp. 30963103, 2004.

[16] K. T. Wong and M. D. Zoltowski, "Self-Initiating MUSICbased direction finding and polarization estimation in spatiopolarizational beamspace," IEEE Transactions on Antennas and Propagation, vol. 48, pp. 1235-1245, 2000.

[17] K. T. Wong and M. D. Zoltowski, "Closed-form direction finding and polarization estimation with arbitrarily spaced electromagnetic vector-sensors at unknown locations," IEEE Transactions on Antennas and Propagation, vol. 48, no. 5, pp. 671-681, 2000.

[18] M. D. Zoltowski and K. T. Wong, "ESPRIT-based 2-D direction finding with a sparse uniform array of electromagnetic vector sensors," IEEE Transactions on Signal Processing, vol. 48, no. 8, pp. 2195-2204, 2000.

[19] M. D. Zoltowski and K. T. Wong, "Closed-form eigenstructure-based direction finding using arbitrary but identical subarrays on a sparse uniform Cartesian array grid," IEEE Transactions on Signal Processing, vol. 48, no. 8, pp. 2205-2210, 2000.

[20] A. N. Lemma, A. J. Van Der Veen, and E. F. Deprettere, "Analysis of joint angle-frequency estimation using ESPRIT," IEEE Transactions on Signal Processing, vol. 51, no. 5, pp. 1264-1283, 2003.

[21] A. N. Lemma, A. J. Van Der Veen, and E. F. Deprettere, "Joint angle-frequency estimation using multi-resolution ESPRIT," in Proceedings of IEEE International Conference on Acoustics, Speech and Signal Processing (ICASSP '98), vol. 4, pp. 19571960, Seattle, Wash, USA, May 1998.

[22] M. D. Zoltowski and C. P. Mathews, "Real-time frequency and 2-D angle estimation with sub-Nyquist spatio-temporal sampling," IEEE Transactions on Signal Processing, vol. 42, no. 10, pp. 2781-2794, 1994.

[23] R. Roy and T. Kailath, "ESPRIT - estimation of signal parameters via rotational invariance techniques," IEEE Transactions on Acoustics, Speech, and Signal Processing, vol. 37, no. 7, pp. 984-995, 1989.

[24] K. T. Wong and M. D. Zoltowski, "High accuracy 2D angle estimation with extended aperture vector sensor arrays," in Proceedings of IEEE International Conference on Acoustics, Speech and Signal Processing (ICASSP '96), vol. 5, pp. 2789-2792, Atlanta, Ga, USA, May 1996.

Fei Ji received the B.S. degree from the Northwestern Polytechnical University in 1992 and the M.S. and Ph.D. degrees from South China University of Technology in 1995 and 1998. Upon graduation, she joined the Department of Electronic Engineering, South China University of Technology in 1998 as a Lecturer. She worked in the City University of Hong Kong as a Research Assistant from March 2001 to July 2002 and a Senior Research Associate from January 2005 to March 2005. She is currently an Associate Professor in the School of Electronic and Information Engineering, South China University of Technology.
Sam Kwong received his B.S. degree and M.A.S. degree in electrical engineering from the State University of New York at Buffalo, USA and University of Waterloo, Canada, in 1983 and 1985, respectively. In 1996, he later obtained his Ph.D. degree from the University of Hagen, Germany. From 1985 to 1987, he was a Diagnostic Engineer with the Control Data Canada where he designed the diagnostic software to detect the manufacture faults of the VLSI chips in the Cyber 430 machine. He later joined the Bell Northern Research Canada as a Member of scientific staff. In 1990, he joined the City University of Hong Kong as a Lecturer in the Department of Electronic Engineering. He is currently an Associate Professor in the Department of Computer Science. 